EPJ Web of Conferences 74, 05001 (2014)

DOI: 10.1051/epjconf/ 20147405001

(C) Owned by the authors, published by EDP Sciences, 2014

\title{
New experiments in gravitational physics
}

\author{
Munawar Karim $^{1 \mathrm{a}}$, Ashfaque H. Bokhari ${ }^{2}$ \\ ${ }^{1}$ Department of Physics, St. John Fisher College, Rochester, NY 14618, USA \\ ${ }^{2}$ Department of Mathematics and Statistics, King Fahd University of Petroleum and Minerals, \\ Dhahran 31261, Saudi Arabia
}

\begin{abstract}
We propose experiments to examine and extend interpretations of the Einstein field equations. Experiments encompass the fields of astrophysics, quantum properties of the gravity field, gravitational effects on quantum electrodynamic phenomena and coupling of spinors to gravity. As an outcome of this work we were able to derive the temperature of the solar corona.
\end{abstract}

\section{Introduction}

New experiments are proposed to extend the interpretations of the Einstein field equations. These experiments are meant to examine the applicability of the field equations to quantum phenomena. Although the experiments are daunting; their results will uncover areas so far unexplored in gravitational physics. We outline four experiments.

\section{Is gravity a quantum field?}

This is an ambitious claim but it may be possible to answer to this question. The proposal is an alteration of the Shapiro time-delay experiment [1]. The Shapiro (excess) time-delay is a consequence of a radio beam travelling along a geodesic of the gravity field of the Sun. There is a continuous, cumulative, time delay as the radio beam traverses a gravity field. Alternatively, one can interpret the time delay as a consequence of discrete scattering of the radio beam off gravity quanta. If gravity is a quantum field one can treat it as a boson gas of discrete quanta. We can estimate properties of the gravitational boson gas outside the surface of the Sun. For any object, such as atoms that constitute the solar corona, which is at equilibrium with the gravity field, its temperature must be equal to the gravity field. This suggests that we treat gravity as a gas, and since it is at equilibrium, as a black body. We can derive conditions for equilibrium and from them the temperature of the gas.

At the surface the gravity field intensity is

$$
\Gamma_{\odot}=\frac{G M_{\odot}}{R_{\odot}^{2}}
$$

The energy density is

\footnotetext{
${ }^{a}$ Corresponding author: karim@sjfc.edu
}

This is an Open Access article distributed under the terms of the Creative Commons Attribution License 4.0, which permits unrestricted use, distribution, and reproduction in any medium, provided the original work is properly cited. 


$$
u=\frac{\Gamma_{\odot}^{2}}{2 G}
$$

Using Stefan-Boltzmann's law the field intensity is

$$
u c=\sigma T^{4}
$$

Whence the equivalent black body temperature at the solar surface is $\approx 10^{7} \mathrm{~K}$. This is consistent with the temperature of the solar corona. If we call $b$ the impact parameter of the radio beam measured from the centre of the Sun then the black body temperature is

$$
T=\left(\frac{G M_{\odot}}{2 b^{4}} \frac{c}{\sigma}\right)=20.5\left(\frac{\sqrt{M_{\odot}}}{b}\right)
$$

The gravity gas temperature varies inversely as the impact parameter $b$. This relationship provides a means to verify the claim that the gravity field behaves as a boson gas.

A black body at $\approx 10^{7} \mathrm{~K}$ has a Boltzmannn factor of

$$
n_{S}=\frac{1}{e^{\hbar \omega / k_{B} T}-1} \gg 1
$$

for radio waves of $20 \mathrm{GHz}$. If gravity is a boson gas its number density will fluctuate as

$$
\frac{\left\langle\left(\Delta n_{S}\right)^{2}\right\rangle}{\left\langle n_{S}^{2}\right\rangle}=\frac{1}{\left\langle n_{S}\right\rangle}+1 \approx 1
$$

Because of the temperature dependence of $n_{s}$ the fluctuations will vary inversely as $b$; these should be so recorded. An inverse relationship would be the consequence of quantum fluctuations of the gravity field.

The scattering geometry is shown below

\section{Dithering image}

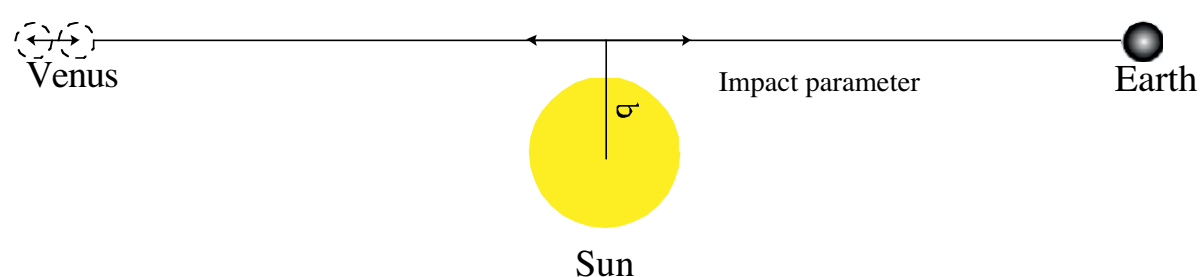

Figure 1. Relative positions of Earth, Sun and Venus for fluctuating Shapiro time delay measurement

Some numbers: for Venus, which subtends a solid angle $4.6 \times 10^{-5}$ radians, a radio antenna of $100 \mathrm{~m}$ diameter will intercept all the reflected waves of frequency $20 \mathrm{GHz}$.

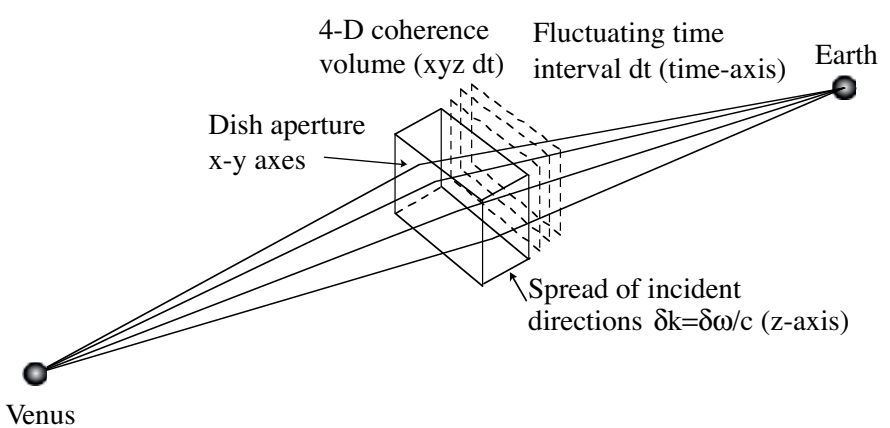


Figure 2. Sketch of fluctuations in coherence volume and scattering geometry

In the proposed experiment the Shapiro time-delay apparatus is used to measure the time-delay of a radio beam reflected off the surface of Venus. The alteration we propose is to measure fluctuations in the time-delay; this would be repeated over a range of the impact parameter. The black body temperature of the gravity field can be extracted from the data. Fluctuations as a function of $b$ would provide evidence of the qunatum nature of the gravity field.

Other sources of fluctuations such as from the solar corona have to be identified and excluded.

\section{Do virtual quantum field excitations follow geodesics?}

Does the stress tensor in the Einstein field equation include energy of the vacuum field, such as the Casimir force, anomalous moment of electrons or the Lamb shift? Are these phenomena altered in a curved metric ? The Casimir force is a pure quantum electrodynamic effect. It is the result of an imbalance between the infinite and continuous vacuum field modes external to a pair of parallel conducting surfaces, and the infinite number of discrete field modes within. The issue of renormalization arises immediately - a dilemma that occurs in several other quantum electrodynamical phenomena such as the anomalous magnetic moment of electrons and the Lamb shift. Although renormalization can be handled in flat space, it is not considered a satisfactory solution, as it requires subtracting divergent functions. Although the cut-off wavelength is conventionally chosen as the Planck length it should be evident that the large energy density at such scales generates a correspondingly large stress tensor. The logical consequence of a large stress tensor is a proportionally large Ricci tensor.

At the Planck length the mass equivalent density of the vacuum field is $\sim 10^{92} \mathrm{gm}_{/} \mathrm{cm}^{3}$. There follow several consequences - all of them absurd.

\subsection{The apparatus}

For the apparatus we choose a modified off-the-shelf product. This is a servo-stabilized Fabry-Perot interferometer etalon built by IC Optical Systems [2]. The interferometer consists of two disks of fused silica, $10 \mathrm{~cm}$ in diameter, $1.8 \mathrm{~cm}$ thick, each polished to $\pm 3 \mathrm{~nm}$ of a flat surface. The surfaces are coated with a gold film of thickness $\approx 1 \mu \mathrm{m}$ with a uniformity of $\pm 2 \mathrm{~nm}$. The root mean square deviation will be smaller. The Yale group [2] was able to attain film uniformity of $\pm 1 \mathrm{~nm}$. If this uniformity can be reproduced in the IOC etalons then one can work the gap down to $\leq 10 \mathrm{~nm}$. At these gaps the Casimir force is a few hundred pounds - a figure that facilitates calibration. The disks are assembled with the gold films facing each other. The etalon is an integrated rigid structure with a high degree of immunity to vibration. It is sealed against the environment. Piezoelectric drivers (PZT) hold the surfaces both at a fixed gap and parallel using a closed-loop feedback system. The PZT's are held under slight compression in order to allow up and down movement. There are 6 capacitors around the edge of the etalon; the pair of X-capacitors measure changes in tilt in the $\mathrm{X}$ direction and the pair of $\mathrm{Y}$-capacitors, measure changes in tilt in the orthogonal direction. There is a fifth capacitor, Z, which detects changes in the plate separation and the last capacitor is a fixed, air spaced reference against which $\mathrm{Z}$ is compared. These pairs are measured in capacitance bridges and any deviation from the user-set position generates an error signal that the servo controller nulls by altering the voltage on the PZT's to maintain a fixed position. This null servo approach gives the accuracy of the system as it does not rely on any reference other than the fixed reference capacitor (which is made of the same materials as the etalon and the other capacitors and for many configurations is largely self-compensating). The servo system linearizes the movement of the PZT's. 
There are three bridges, two for parallelism changes and one for mean gap changes. Gaps can be maintained to within $\pm 10^{-12} \mathrm{~m} / \mathrm{Hz}^{1 / 2}$; the surface parallelism to within $\pm 10^{-11} \mathrm{rads} / \mathrm{Hz}^{1 / 2}$. Reducing the bandwidth yields a gap uncertainty to within $\pm 3 \times 10^{-15} \mathrm{~m}$.

When brought in close proximity $(\approx 1 \mu \mathrm{m})$ the Casmir force will attract the disks. As the gap between the plates is reduced the Casimir force increases. Once a gap is selected the feedback servo reacts to keep the gap fixed by applying a compensating force on the PZT drivers until it is equal to the Casimir force. Measuring the Casimir force entails measuring the counter force on the plates exerted by the PZT drivers.

\subsection{Calibration}

Since the PZT drivers are integrated, rigid stacks, force calibration needs to be indirect. The method we have devised calibrates the drive voltage applied to the PZT's since the force exerted varies as the voltage (the closed-loop feedback linearizes the voltage-force response). With the F-B plates at a fixed gap and resting on a horizontal platform, known weights are placed on the upper surface. This causes the gap to shrink initially; an error signal is generated. In order to maintain the gap the feedback circuit increases the force on the PZT's by increasing the drive voltage until the error signal is zeroed. The drive voltage versus weight is recorded for the chosen gap. The drive voltage is plotted against the weights. Since the feed back servo linearizes the PZT response and removes the hysteresis, the voltage vs. weight graph will be linear. For the etalons in question the linearity/hysteresis precision is within $\pm 0.49 \mathrm{~nm}$; it can be reduced to $\pm 0.14 \mathrm{~nm}$ using a 16-bit interface. Extrapolating the graph to zero weight gives the voltage that corresponds to the net force the PZT must exert to maintain the particular gap between the plates.

The steps are repeated for different gaps. Forces versus voltage graphs are drawn for each gap. A new calibration graph is obtained by re-arranging the figures for force versus gap from force versus voltage. The force vs. gap is linear because the relation between capacitance and gap is linear. The force vs. gap relation is the desired calibration of the PZT drivers.

The experiment then consists of choosing a gap, determining the force between the surfaces from the force vs. gap calibration. Then repeating the steps for decreasing gaps. As the gap decreases below $1 \mu \mathrm{m}$ the Casimir force contribution will increase rapidly. Plotting the force against $1 / d^{4}$ may identify its magnitude. If it is the Casimir force the graph should be a straight line with a slope of $-\pi^{2} \hbar c / 240$.

\subsection{Sensitivity}

Using etalons what is the expected precision of the Casimir force? PZT voltages are in the range of 700 volts. Using precision digital voltmeters, voltages can be measured to $8 \times 10^{-4} \mathrm{~V} / \mathrm{Hz}^{1 / 2}$ or a few parts in $10^{6}$. This assumes that high frequency fluctuations in the voltage will be filtered out. One can do better by using the dielectric/elastic properties of one of the PZT segments as a capacitor in an ACbridge. Compression or expansion of the PZT stack alters the thickness of each of the PZT segments, in turn altering the capacitance of the segments. One can relate the change in the capacitance to the force exerted by the PZT. Since we are dealing with piezo materials the AC excitation will also affect the thickness, and hence the capacitance, of the segment in question. The thickness can be kept constant by applying an equal excitation of opposite polarity to neighboring segments. By repeating the measurement for decreasing excitation voltages and extrapolating to zero voltage one can isolate the contribution of the excitation-voltage induced change in the capacitance of the selected segment. If we choose the capacitor method the sensitivity is limited either by the $\tan \delta$ of the PZT material $\approx 20 \times 10^{3}$; if one can substitute sapphire for one of the segments in the PZT stack then the $\tan \delta$ is 
reduced to $10^{-4}$ which translates to Johnson noise of $5.7 \times 10^{-9} \mathrm{~V} / \mathrm{Hz}^{1 / 2} @ 1 \mathrm{kHz}$, or the noise of the following op-amp $\approx 5 \times 10^{-9} \mathrm{~V} / \mathrm{Hz}^{1 / 2}$. If it is the latter then the uncertainty is $\pm 5 \times 10^{-12} / \mathrm{Hz}^{1 / 2}$. Yet another method is to convert the change in capacitance to a change in frequency by using the capacitor as a resonant element in an LCR circuit. The frequency uncertainty is limited by $\tan \delta$ to $\approx \delta v / v=Q^{-1} \approx 5 \times 10^{-6} @ 9 \mathrm{GHz}$. Thus $\delta v \leq 5 \times 10^{4} \mathrm{~Hz}$. So either way the noise spectral density is between $\pm 10^{-9} \mathrm{~V} / \mathrm{Hz}^{1 / 2}$ to $\pm 10^{-11} \mathrm{~V} / \mathrm{Hz}^{1 / 2}$. With reasonable integration intervals the minimum sensitivity is between 1 part in $10^{9}$ to $10^{11}$. Even lower sensitivities can be obtained by using Josephson junction voltmeters or Single Electron Transistor amplifiers but with the added cost and complexity of a cryogenic environment.

The Casimir disks form two arms of an AC-bridge. The output is fed into a phase-sensitive detector. There is a $2 \mathrm{~Hz}$ reference oscillator. Amplifier noise is $\pm 5 \mathrm{nV} / \mathrm{Hz}^{1 / 2}$, the band-width is $10^{-2} \mathrm{~Hz}$. The expected sensitivity is 5 parts in $10^{10}$.

Noise can be further suppressed. Using the theory of partial coherence (Hanbury-Brown Twiss effect), the output is fed into a circuit shown in Fig. 3. The signal is injected into a band-pass filter which is centered at the frequency of the signal. It is then split into two branches one of which is delayed with respect to the other. The two branches are multiplied $(\Delta V(t+\tau) \Delta V(t))$ then integrated over a time interval $T$ (inverse of the band-width). The output is the mutual coherence function

$$
\left\langle\Delta I_{1}(t+\tau) \Delta I_{2}(t)\right\rangle_{T}=\left|\tilde{\Gamma}_{12}(\tau)\right|^{2}
$$

The delay interval $\tau$ is adjsuted until $\left|\tilde{\Gamma}_{12}(\tau)\right|^{2} \rightarrow 0$. Noise in the output is reduced as a consequence by $10^{5}$. For details see $[4,5]$. Altogether the sensitivity should be a few parts in $10^{15}$.

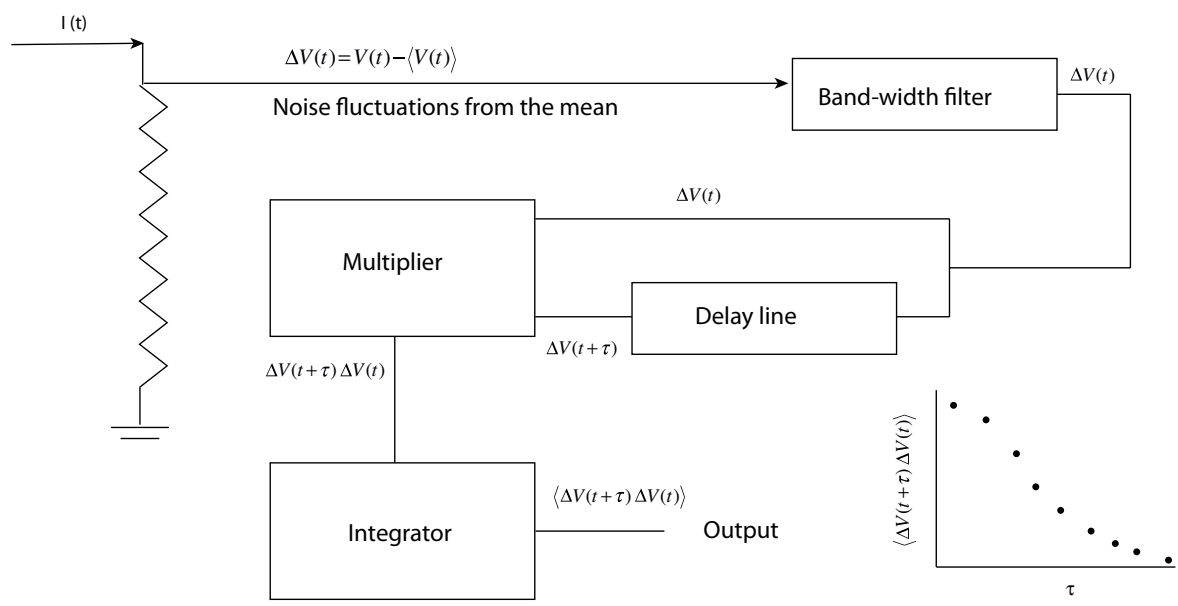

Figure 3. Circuit for noise cancellation utilizing the Hanbury-Brown Twiss effect. 


\subsection{Method of Measurement}

The Casimir force/area between plane conductors separated by a gap $d$ is $F=-\pi^{2} \hbar c / 240 d^{4}$. The first part of the experiment will be to establish that the attraction between the plates is the Casimir force by confirming the $1 / d^{4}$ dependence of the force. This requires setting the gaps between the plates between 100 to $1000 \mathrm{~nm}$. For a chosen gap the force is obtained from the force vs. gap calibration data. The measurement is repeated throughout the range of gaps. Force is plotted against $1 / d^{4}$. If the data points fall on a straight line then the force can be identified as the Casimir force. The slope should be $-\pi^{2} \hbar c / 240$. Contact potentials between circuit elements and patch effects on the films can mask the $1 / d^{4}$ dependence; these aberrations must be taken care of by judicious application of compensating potentials.

\subsection{Systematic Uncertainties}

With changing orientation the etalon chasses will also be affected by the Ricci tensor. Keeping the angle fixed and measuring the force of attraction isolates this effect. Plotting the force at each angle against $1 / d^{4}$ and extrapolating to $1 / d^{4} \rightarrow 0$ distinguishes the Casimir force from the tidal force acting on the etalons. Or fit the data to a polynomial in powers of $1 / d$ and extrapolate the result in the limit as $1 / d \rightarrow 0$. The latter option covers the possibility that the Casimir force may not follow a $1 / d^{4}$ dependence under the influence of curvature. The electronic drift of the etalons is $\pm 50 \mathrm{pm} /{ }^{\circ} \mathrm{C}-$ with careful control the temperature stability is 1 part in $10^{10}$.

Distortions due to the weight of the apparatus can be expected as it is rotated about its symmetry axis. They can be minimized by floating the apparatus in a temperature-controlled bath in a liquid of density equal to the etalons $\left(\approx 2.5 \mathrm{~g} / \mathrm{cm}^{3}\right)$. Liquids of calibrated densities are available with uncertainties of $\pm 0.005 \mathrm{~g} / \mathrm{cm}^{3}$. Neutral buoyancy will cancel effects of weight. Any asymmetry between two plates (for example a slight inequality in the weights of two plates) will occur at the frequency of rotation; different from the signal which will occur at twice the frequency. These extraneous effects necessarily occur when the apparatus is located on Earth. Such effects would disappear if the apparatus is in free fall, but the cost and complications of a space-based experiment is not an option. It is understood that environmental conditions such as pressure and temperature are carefully controlled and are not an issue.

This is a null experiment. The disks are arranged so that each pair is sensitive only to the $R_{r}^{r}$ or $R_{\theta}^{\theta}$ components of the Ricci tensor. The output is sensitive only to the difference in the effect on the Casimir force between the two components of the Ricci tensor. The Ricci tensor is on the surface of Earth. 


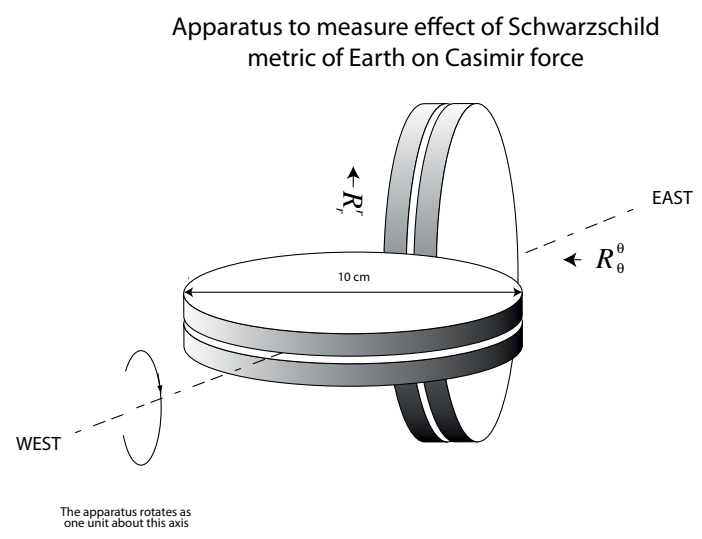

Figure 4. Orthogonal pairs of Casimir plates arranged to detect effect of radial and angular Ricci tensors

\subsection{Theoretical Estimates}

Although it is not known if the Casimir force is affected by curvature we can estimate the magnitude if it does. We use an expression of the Casimir force as an expectation value of the stress tensor [1]. It has been renormalized.

$$
\left\langle T^{\mu v}\right\rangle=\frac{\pi^{2} \hbar c}{180 a^{4}}\left[\frac{1}{4} \eta^{\mu v}-\hat{x}^{\mu} \hat{x}^{v}\right]
$$

$\eta^{\mu v}$ is the Minkowski metric and $\hat{x}^{\mu} \hat{x}^{v}$ are area normals of the plates. Since $\left\langle T^{\mu v}\right\rangle$ is a traceless tensor when we insert it in the Einstein equation it looks like this

$$
R^{\mu v}=-\frac{8 \pi G}{c^{4}}\left\langle T^{\mu v}\right\rangle
$$

When the right hand side is zero the vacuum solution is the Schwarzschild metric. $\left\langle T^{\mu v}\right\rangle$ is very small so we can use the Schwarzschild metric as a first order solution. The second order term is a small correction term

$$
g^{\mu v}=g_{S}^{\mu v}+\varepsilon \gamma^{\mu v}
$$

$\varepsilon \gamma^{\mu \nu}$ is a small time-independent perturbation due to the presence of the stress tensor and is expected to be zero far from the stress tensor. $g_{s}^{\mu v}$ is the Schwarzschild metric. We use the perturbed metric (1.10) to calculate $\gamma^{\mu v}$ using the known form of the stress tensor in (1.8), keeping terms to first order in $\varepsilon$ and in $m / r$ also. $\gamma^{\mu v}$ are potentials generated by the stress tensor. $\gamma^{\mu v}$ is then inserted in the unperturbed stress tensor. At the end we get a perturbed stress tensor. To first order the solution for $\gamma^{\mu v}$ is obtained from the Poisson equation.

$$
\varphi=-\frac{4 \pi G}{c^{2}} \int \frac{\rho\left(x^{\prime}\right)}{\left|x-x^{\prime}\right|} d v^{\prime}
$$




$$
\gamma^{\mu v}=-\frac{4 \pi G}{c^{4}} \frac{\left\langle T^{\mu v}\right\rangle v}{r}
$$

where the source term is just the energy content of $\left\langle T^{\mu v}\right\rangle$ and $v=7.85 \times 10^{-9} \mathrm{~m}^{3}$ which is the volume of the cavity between the Casimir plates. The perturbed metric is thus

$$
g^{\mu v}=g_{s}^{\mu v}-\varepsilon \frac{4 \pi G}{c^{4}} \frac{\left\langle T^{\mu v}\right\rangle v}{r}
$$

The stress tensor is altered to

$$
\left\langle T^{\mu v}\right\rangle_{G}=\frac{\pi^{2} \hbar c}{180 a^{4}}\left[\frac{1}{4}\left(g_{S}^{\mu v}-\frac{4 \pi G}{c^{4}} \frac{\left\langle T^{\mu v}\right\rangle_{v}}{r}\right)-\hat{x}^{\mu} \hat{x}^{v}\right]
$$

This then is the altered Casimir force.

\section{Spinors in curved metric}

Spin half particles such as electrons are described by spinors. Unlike ordinary objects spin half particles must be rotated by $4 \pi$ rather than $2 \pi$ before they return to their original state. Electrons have a g-factor of 2 . Even in flat space, vacuum polarization effects increase the $\mathrm{g}$-factor to

$2(1+\alpha / 2 \pi) \approx 2.0023$, with the implication that a spin half particle must rotate by slightly more than

two turns before returning to its original state. Spinors follow a geometry different from other objects. Gravity has its own geometry; an angle defect arises when a vector is parallel transported around a closed loop in the gravity field of a mass. This is a consequence of the curved geometry of space-time. This is called a geodetic effect. The most common demonstration of this phenomenon is the precession of a spinning mass (gyroscope) in a gravity field. One may ask if there is a geodetic effect when the spinning objects are electrons (spinors)? If instead of a vector, a spinor is parallel transported around a closed loop in the presence of a massive object will it too display an angle defect analogous to a mechanical gyroscope? There is no apriori reason to believe that it must be so.

Although this effect may be inconsequential under ordinary circumstances where curvature effects are miniscule, the effect may be significant for strong gravity fields such as for electrons in white dwarfs or neutrons in neutron stars. Quite possibly the equation of state for electrons in white dwarfs and neutrons in neutron stars may be altered. If so the effect will change the pressure of a degenerate spinor gas thus affecting stability conditions of such stars.

In order to answer this question we propose an experiment. We will examine the coupling of a spinor to a gravity field - look for an angle defect on an electron spinor. For the spinor we propose using a spin-polarized material - Ni's ferrimagnetic alloy - $D y_{6} F_{23}[6]$ in the shape of a cylinder. At the temperature where the magnetization compensation point is reached there is complete cancellation of spin and orbital angular momenta. The object has zero magnetic moment, although the spins are aligned. This occurs at room temperature. At this temperature $\approx 10^{22}$ electron spins are aligned. The cylinder is suspended horizontally from a torsion wire on the South Pole. At the South Pole the angular momentum of the spinor gyro is immune to precession caused by Earth's rotation. The apparatus is mounted on a horizontal turntable. A telescope points to the star Canopus which is used as a reference point. As Earth spins the turntable remains locked to Canopus. The scheme is based on Braginsky/Polnarev/Thorne's proposed experiment [7].

This arrangement decouples the platform from Earth's rotation. The spin axis is perpendicular to the axis of rotation of Earth - it is decoupled from the angular momentum of Earth. A weak magnetic field oscillating@1 kHz exerts a compensating force on the cylinder [8] (the oscillating magnetic field exerts a repulsive force on either side of the cylinder because of the diamagnetic property 
of $\left.D y_{6} \mathrm{Fe}_{23}\right)$. The force is immune to ambient electric or magnetic fields. The force is calibrated against the deflection angle of the spin-polarized cylinder.

The spinor gyro is parallel transported as Earth orbits the Sun. If the spinor gyro couples to the (Schwarzschild) metric of the Sun the axis will deviate from its initial orientation (defined by the alignment with Canopus). This will generate an error signal. The error signal drives a compensating force in a direction to null the error signal. The compensating force is recorded over a period of a year. We look for a one-year period in the compensating force. The amplitude of the force is a measure of the geodetic effect of the electron spinor in the Schwarzschild metric of the Sun.

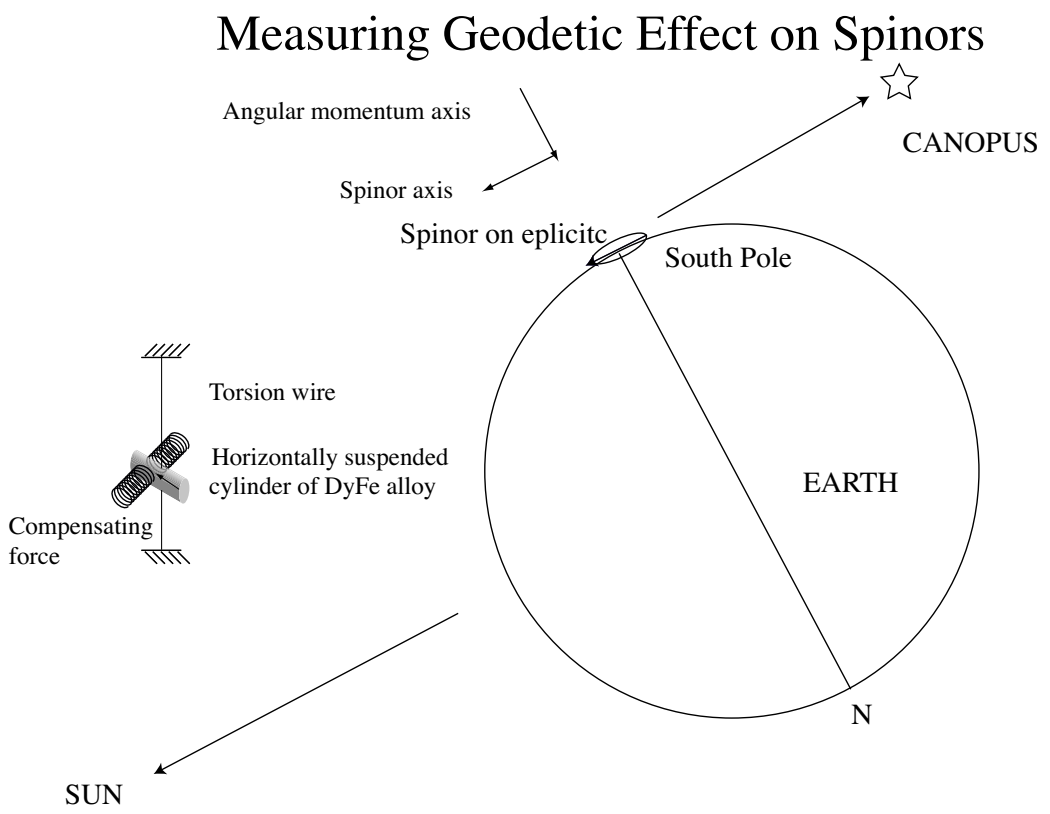

Figure 5. Location and orientation of spinor gyro on the South Pole - referred to the star Canopus.

For a mechanical gyro orbiting the Sun at $1 \mathrm{AU}$ (located on Earth) the angle defect for an orbit is $3 \pi M_{\odot} / R \approx 10^{-7}=0.122$ arc seconds. If a spinor gyro accumulates a similar angle defect one needs to measure this deflection against disturbances such as seismic noise, which for a stiff suspension is about $10^{-8} \mathrm{~cm} / \mathrm{Hz}^{1 / 2}$ at the South Pole, suggesting that the turntable will have to be isolated against terrestrial vibrations. Using a feedback loop the temperature can be kept to within $\pm 10^{-3} \mathrm{C}^{\circ}$ of the compensation temperature. Fluctuations in the atmosphere can blur the position of the reference star by $<0.004$ " (7). Observing the star at multiple wavelengths can reduce this source of uncertainty.

\section{Experiment to distinguish between red shift and Doppler shift}

Often distances are measured using Hubble's Law. This requires measuring the Doppler shift of spectral lines. However, the Doppler effect and/or a gravitational red/blue shift can both displace spectral lines. If the captured light has travelled through a gravitational field, for example through a sea of gravitational waves of cosmological origin, then there will be an ambiguity in the interpretation of the detected spectral shift. Since both Doppler and gravitational spectral shifts are wavelength independent it would appear that it is not possible to separate the two effects. 
However, the width of the spectral lines is broadened due to scattering by the gravitational field much like scattering from random media [5]. An example is shown in Figure 6 [9].

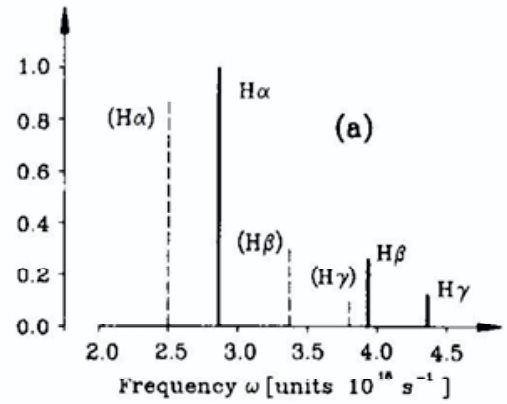

Figure 6. (a) Peaks red-shifted

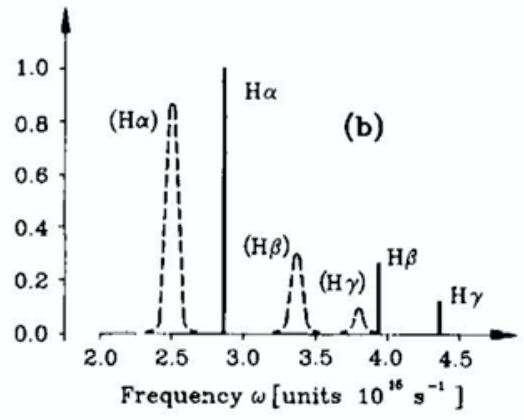

(b) Peaks red-shifted and broadened

Doppler or gravitational red-shift can be distinguished by measuring both the spectral shift as well as peak broadening. Whereas Doppler effect shifts only the wavelength of spectral lines, gravitational effect shifts both the spectral lines and broadens them. The line broadening is the consequence of a light beam that can take multiple paths as it traverses a sea of gravitational waves because the waves are oscillating.

Stellar sources are usually moving at high velocities so it is difficult to get an accurate measure of the line-widths. However with quality Fabry-Perot etalons line widths as narrow as Doppler broadened peaks from sources moving at $10 \mathrm{~km} / \mathrm{s}$ can be discriminated [2].

Measurements can be repeated for several peaks. The slope of a graph of $Q=\omega / \Delta \omega_{0}$ vs. the central frequency $\omega$ provides information on the 2-point correlation function of the scattering field. Using this method the effects of the Doppler shift can be distinguished from gravitational red shift.

\section{Conclusion}

We have described four experiments that extend the applicability of the Einstein field equations to new arenas. Although some of the proposed experiments are more developed than others, each one of them explores novel phenomena. Some of the ideas raised are speculative, but speculation is the door to new frontiers; the suggested experiments are meant to stimulate a search for evidence that will either confirm or refute them.

\section{References}

1. L.S. Brown and G.J. Maclay, Phys. Rev. 184, 1272 (1969)

2. IC Optical Systems Ltd., Beckenham, Kent, BR3 4TW, UK

3. A.O. Sushkov, W.J. Kim, D.A.R. Dalvit, S.K. Lamoreaux, Nature Phys. 7, 230 (2011)

4. M.Karim, http://dl.dropboxusercontent.com/u/39112874/NoiseSupression.docx

5. Optical Coherence and Quantum Optics, L. Mandel and E. Wolf, (Cambridge U.P. 1995)

6. W-T Ni, Proceedings of the Fourth Marcel Grossmann Meeting on General Relativity, R. Ruffini (ed.), Elsevier Science Publishers B.V., 1335 (1992), and Rep. Prog. Phys. 73, 1, (2010)

7. V.B. Braginsky, A.G. Polnarev, K. Thorne, Phys. Rev. Lett. 53, 863 (1984)

8. M. Karim, W.J. Toohey, Am. J. Phys. 11, 1043 (1986)

9. D.F.V. James and E.Wolf, Phys. Lett. A 146, 167 (1990) 